\title{
APPROXIMATION OF ANALYTIC FUNCTIONS ON COMPACT SETS AND BERNSTEIN'S INEQUALITY
}

\author{
BY \\ M. S. BAOUENDI( 1 ) AND C. GOULAOUIC
}

\begin{abstract}
The characterization of analytic functions defined on a compact set $K$ in $\mathbf{R}_{N}$ by their polynomial approximation is possible if and only if $K$ satisfies some "Bernstein type inequality", estimating any polynomial $P$ in some neighborhood of $K$ using the supremum of $\boldsymbol{P}$ on $\boldsymbol{K}$. Some criterions and examples are given. Approximation by more general sets of analytic functions is also discussed.
\end{abstract}

0. Introduction. Let $K$ be a compact set in $\mathbf{R}^{N}$. It is known [1] that the distance of any analytic function defined on $K$ to the space of polynomials of degree $n$ is exponentially decreasing as $n$ becomes large.

We say that $K$ satisfies (A) if, conversely, any continuous function, whose distance to the space of polynomials of degree $n$ is exponentially decreasing, is necessarily analytic on $K$.

We discuss here the question: What are the compact sets in $\mathbf{R}^{\mathbf{N}}$ which satisfy (A)?

S. Bernstein proved in 1912 [2] that the interval $[-1,+1]$ satisfies (A). (See also [5] and [6] for the one dimensional complex case.) He used the so-called Bernstein's inequality, estimating any polynomial $P$ in the complex plane by means of the supremum of $|P|$ on $[-1,+1]$.

We prove here that (A) holds for $K$ if and only if $K$ satisfies a "Bernstein (or Markov) type inequality", which gives a precise estimate of any polynomial $\boldsymbol{P}$ on some neighborhood of $K$ using the supremum of $P$ on $K$.

This characterization yields to some positive criterions (in order that $K$ satisfy (A)), and also some negative examples.

We show in particular that some compact sets, for which the characterization of $C^{\infty}$ functions by their polynomial approximation is not possible, do satisfy (A). (See [7] for the $C^{\infty}$ case.)

In part I, we discuss the approximation of analytic functions defined on compact sets by more general sets of analytic functions than polynomials. This may be used, for example, in the case of the approximation by the eigenvectors of some differential operators. We do not give here this application.

In part II, we restrict ourselves to the polynomial case and we give some criterions, positive and negative examples.

Received by the editors November 15, 1972.

AMS (MOS) subject classifications (1970). Primary 41A10, 26A90; Secondary 4IA25, 26A75.

Key words and phrases. Approximation of real-analytic functions, polynomials. Bernstein inequality.

(I) The first author was partially supported during this work by NSF Grant GP 35825. 


\section{APPROXIMATION BY A SET OF ANALYTIC FUNCTIONS}

We introduce first some notations and definitions.

Let $K$ be a compact set in $\mathbf{R}^{N}$. We denote by $C(K)$ the space of complex valued continuous functions defined on $K$, equipped with the uniform convergence norm.

The real space $\mathbf{R}^{N}$ is embedded in the complex space $\mathbf{C}^{N}=\mathbf{R}^{N}+i \mathbf{R}^{N}$, as usual. We consider $K$ as a compact set in $\mathbf{C}^{N}$.

Let $\Omega$ be an open set in $\mathbf{C}^{N}$ and $O(\Omega)$ the Banach space of holomorphic and bounded functions defined in $\Omega$, provided with the uniform convergence norm.

Let $\mathcal{O}(K)$ be the space of holomorphic germs defined on $K$. We equip $\mathcal{O}(K)$ with the inductive limit topology of $\mathcal{O}(\Omega)$ for all $\Omega$, where $\Omega$ is an open neighborhood of $K$ in $\mathbf{C}^{N}$. (We may restrict ourselves to the sets $\Omega$ such that any component of $\Omega$ intersects $K$.)

We denote by $\mathcal{N}_{K}(\Omega)$ the kernel of the natural restriction $r_{\Omega}: \mathcal{O}(\Omega) \rightarrow C(K)$, and by $\mathcal{A}_{\Omega}(K)$ its range provided with the quotient topology $\mathcal{O}(\Omega) / \mathcal{N} \mathcal{N}_{K}(\Omega)$.

The mappings $r_{\Omega}$ define the restriction $r, r: \mathcal{O}(K) \rightarrow C(K)$. The kernel of $r$ is

$$
\mathcal{N}(K)=\operatorname{ind}_{K \subset \Omega} \lim _{K}(\Omega)
$$

and its range is the space of analytic functions defined on $K$

$$
\mathcal{A}(K)=\operatorname{ind}_{K \subset \Omega} \lim _{\Omega}(K) .
$$

Let us observe that if $\mathcal{N}(K)=\mathcal{N}_{\Omega}(K)=0$, the mapping $r$ is injective and $\mathcal{A}(K)$ is isomorphic to $\mathcal{O}(K)$. This condition is satisfied if and only if no component of $K$ is contained in an analytic set of $\mathbf{R}^{N}$.

Now, let us consider a sequence $\left(H_{n}\right)$ of finite dimensional vector spaces satisfying

$$
H_{0} \subset H_{1} \subset \cdots \subset H_{n} \subset H_{n+1} \subset \cdots \subset \mathcal{O}(K) \text {. }
$$

Let $\left(\mu_{n}\right)$ be an increasing sequence of strictly positive real numbers such that

$$
\text { for any } a \in] 0,1\left[, \quad \sum_{n=0}^{\infty} a^{\mu_{n}}<+\infty\right. \text {. }
$$

We introduce the following properties related to the compact $K$ and the sequences $\left(H_{n}\right)$ and $\left(\mu_{n}\right)$.

Property (A): analytic approximation. Any function $f \in C(K)$, for which there exists $C>0$ and $a \in] 0,1[$ such that $(2)$ for each $n \in \mathbf{N}$

$$
d_{K}\left(f, r\left(H_{n}\right)\right) \leq C a^{\mu_{n}}
$$

belongs to $A(K)$.

(2) If $f \in C(K)$ and $F$ is a subspace of $C(K)$ we denote $d_{K}(f, F)=\inf _{g \in F} \sup _{x \in K}|f(x)-g(x)|$. 
Property (B): Bernstein's inequality. For any real number $b>1$, there exists an open set $\Omega$, neighborhood of $K$ in $C^{N}$, such that, for any $P \in H_{n}$, there exists $Q \in \mathcal{N}(K)$ satisfying

$$
P+Q \in \mathcal{O}(\Omega) \text { and } \sup _{x \in \Omega}|P(x)+Q(x)| \leq b^{\mu_{n}} \sup _{x \in K}|P(x)|
$$

We have the following result:

Theorem (I.1). Let $K$ be a compact set in $\mathbf{R}^{N}$, and $\left(H_{n}\right)$ and $\left(\mu_{n}\right)$ satisfy (I.3) and (I. 4). The properties (A) and (B) are equivalent.

Proof. 1. (B) implies (A). We assume (B). Let $f \in C(K)$ satisfy (1.5). We shall prove that $f \in \mathcal{A}(K)$.

Let $P_{n} \in H_{n}$ such that $d_{K}\left(f, r\left(H_{n}\right)\right)=\sup _{x \in K}\left|f(x)-P_{n}(x)\right|$. Since we have

$$
\sup _{x \in K}\left|P_{n}(x)-P_{n-1}(x)\right| \leq 2 d_{K}\left(f, r\left(H_{n-1}\right)\right) \leq 2 C a^{\mu_{n-1}},
$$

and using (I.4), the series $P_{0}+\sum_{n=1}^{\infty}\left(P_{n}-P_{n-1}\right)$ is convergent in $C(K)$, its sum is $f$.

We choose $b>1$ such that $a b<1$, and let $Q_{n}$ be the element of $\propto N(K)$ given by (B) and associated to $P_{n}-P_{n-1}$. The series $P_{0}+Q_{0}+\sum_{n=1}^{\infty}\left(P_{n}-P_{n-1}+Q_{n}\right)$ coincides with $f$ on $K$ and is convergent in $\mathcal{O}(\Omega)$, since by virtue of (B) we have

$$
\sup _{x \in \Omega}\left|P_{n}(x)-P_{n-1}(x)+Q_{n}(x)\right| \leq 2 C a^{\mu_{n-1}} b^{\mu_{n-1}} \text {. }
$$

We have shown that $f$ is in $\mathscr{A}(K)$ and that property (A) holds.

2. (A) implies (B). First of all, it is easy to see that property (B) is equivalent to the following two properties:

(I.7) There exists an open set $U$, neighborhood of $K$ in $\mathbf{C}^{N}$, such that for any $n \in \mathbf{N}, r\left(H_{n}\right) \subset \mathcal{A}_{U}(K)$ (i.e. $H_{n} \subset \mathcal{O}(U)+\mathcal{N}(K)$ ).

(I.8) For any real number $b>1$, there exists an open set $\Omega$, neighborhood of $K$ in $\mathbf{C}^{N}, \Omega \subset U\left(U\right.$ is given in (I.7)) such that for any $P \in H_{n}$

$$
\|r(P)\|_{A_{\Omega}(K)} \leq b^{\mu_{n}}\|r(P)\|_{C(K)} .
$$

We prove first that (A) implies (I.7). For $a \in] 0,1[$, let us consider the space

$$
E_{a}=\left\{f \in C(K) ; \sup _{n}\left(a^{-\mu_{n}} d_{K}\left(f, r\left(H_{n}\right)\right)\right)<+\infty\right\} \text {. }
$$

It is a Banach space with the norm

$$
\|f\|_{E_{a}}=\|f\|_{C(K)}+\sup _{n}\left(a^{-\mu_{n}} d_{K}\left(f, r\left(H_{n}\right)\right)\right) .
$$

The space $E=$ ind $\lim _{0<a<1} E_{a}$ is continuously embedded in $C(K)$.

We observe that property $(A)$ is equivalent to the following one:

$$
E \subset \mathcal{A}(K) \text {. }
$$


It follows from the closed graph theorem and property $(A)$ that the injection (I.9) is continuous. Using a well-known theorem about continuous linear mappings in inductive limits of Fréchet spaces (see [3] for example), we can state that for any $a \in] 0,1\left[\right.$, there exists an open set $U$, neighborhood of $K$ in $\mathbf{C}^{N}$, such that

$$
E_{a} \subset \mathcal{A}_{U}(K)
$$

with continuous injection.

Property (I.7) follows readily from (I.10) since for any $n \in \mathbf{N}$ and any $a \in] 0,1\left[\right.$ we have $r\left(H_{n}\right) \subset E_{a}$.

Now we have to show that (A) implies (I.8). We prove it by contradiction. We assume (A) (and therefore (I.7)) and we suppose that (I.8) is not true.

Let $\left(\Omega_{k}\right), k \in \mathrm{N}$, be a fundamental system of open neighborhoods of $K$ in $\mathbf{C}^{N}$, decreasing and contained in $U$ (U being given in (I.7)). Since (I.8) is false, there exists $b>1$ such that, for any $k \in \mathrm{N}$, there exists $n_{k}$ and $P_{n_{k}} \in H_{n_{k}}$ satisfying

$$
\left\|r\left(P_{n_{k}}\right)\right\|_{\mathcal{A}_{B_{k}}(K)}>b^{\mu_{n_{k}}}\left\|P_{n_{k}}\right\|_{C(K)}
$$

Without loss of generality we may assume $\left\|P_{n_{k}}\right\|_{C(K)}=1$ and the sequence $n_{k}$ is strictly increasing. [Indeed, it is easy to see, since $H_{n}$ is a finite dimensional space, that (I.8) would be true if we restrict it to a fixed $H_{n_{0}}$.]

For $a \in] 0,1[$, we denote

$$
\begin{aligned}
& \mathcal{E}_{a}=\left\{\left(c_{n}\right) \in \mathbf{C}^{\mathbf{N}} ;\left\|c_{n}\right\|_{a}=\sup _{n}\left|c_{n} a^{-\mu_{n}}\right|<\infty\right\}, \\
& \varepsilon=\operatorname{ind}_{0<a<1} \lim _{a},
\end{aligned}
$$

provided with their natural topology. For $\left(c_{n}\right) \in \varepsilon_{a}$ we have

$$
\left\|\sum_{k} c_{n_{k}} P_{n_{k}}\right\|_{C(K)} \leq\left\|\left(c_{n}\right)\right\|_{b} \sum_{k} a^{m_{n_{k}}} .
$$

Therefore the linear mapping

$$
\left(c_{n}\right) \mapsto \sum_{k} c_{n_{k}} P_{n_{k}}=u\left(\left(c_{n}\right)\right)
$$

is continuous from $\varepsilon$ into $C(K)$. Let us prove that its range is in $\mathcal{A}(K)$, which implies, using the closed graph theorem, that $u$ is a continuous mapping from $\varepsilon$ into $\mathcal{A}(K)$.

Let $\left(c_{n}\right) \in \varepsilon_{a}$. In order to prove that $u\left(\left(c_{n}\right)\right)$ is in $\mathcal{A}(K)$, it is sufficient to prove (using (A)) that there exist $\left.a_{1} \in\right] 0,1[$ and $C>0$ such that

(I.12) For any $l \in \mathbf{N}, d_{K}\left(U\left(\left(c_{n}\right)\right), r\left(H_{l}\right)\right) \leq C a_{1}^{\mu_{1}}$.

Indeed, we have

$$
d_{K}\left(U\left(\left(c_{n}\right)\right), r\left(H_{n_{j}}\right)\right) \leq\left\|\sum_{k=j+1}^{\infty} c_{n_{k}} P_{n_{k}}\right\| \leq\left\|\left(c_{n}\right)\right\|_{k} \sum_{k=j+1}^{\infty} a^{m_{n_{k}}} .
$$


If $a<a_{1}<1$, we obtain

$$
d_{K}\left(u\left(\left(c_{n}\right)\right), r\left(H_{n_{j}}\right)\right) \leq C a_{1}^{\mu_{n+1}} \leq C a_{1}^{\mu_{n}}
$$

with $C=\left\|\left(c_{n}\right)\right\|_{a} \cdot \sum_{n}\left(a / a_{1}\right)^{m_{n}}$. On the other hand, we have for $n_{j}<q<n_{j+1}$

$$
\begin{aligned}
d_{K}\left(\mathcal{U}\left(\left(c_{n}\right)\right), r\left(H_{q}\right)\right) & \leq d_{K}\left(U\left(\left(c_{n}\right)\right), r\left(H_{n_{j}}\right)\right) \\
& \leq C a_{1}^{\mu_{n+1}} \leq C a_{1}^{\mu_{\varphi}},
\end{aligned}
$$

which ends the proof of (I.12).

We are going to prove now that the continuity of $u$ from $\varepsilon$ into $\mathscr{A}(K)$ implies a contradiction with (I.11).

Using a well-known theorem (see the first part of the present proof), we can say that for any $a \in] 0,1[$, there exist $p \in \mathbf{N}$ and $L>0$ such that, for each $n \in \mathbf{N}$,

$$
\left\|u\left(\left(c_{n}\right)\right)\right\|_{A_{\Omega_{1}}(K)} \leq L\left\|\left(c_{n}\right)\right\|_{\text {a }}
$$

We choose $a$ such that $a b>1$, and $k$ large enough in order to get

$$
L \leq(a b)^{\mu_{n_{k}}} \text { and } \Omega_{k} \subset \Omega_{p}
$$

( $L$ and $\Omega_{p}$ being given in (I.13) with the choice we have done for $a$ ).

Let us put, in (I.13), $c_{n}=0$ for $n \neq n_{k}$ and $c_{n_{k}}=1$; we obtain

$$
\left\|r\left(P_{n_{k}}\right)\right\|_{A_{B_{p}}(K)} \leq L a^{-\mu_{n_{k}}} \text {, }
$$

and using (I.14)

$$
\left\|r\left(P_{n_{k}}\right)\right\|_{A_{\Omega_{k}(K)}} \leq\left\|r\left(P_{n_{k}}\right)\right\|_{A_{Q_{j}}(K)} \leq L a^{-\mu_{n_{k}}} \leq b^{\mu_{n_{k}}} .
$$

This gives us a contradiction with (I.11) and ends the proof of Theorem (I.1).

We introduce now another property :

Property (M): Markov's inequality. For any $b>1$ there exists $C>0$ such that for any $P \in H_{n}$ there exists $Q \in \mathcal{N}(K)$ satisfying for each $\alpha \in \mathbf{N}^{N}(3)$

$$
\sup _{x \in K}\left|D^{\alpha}(P+Q)(x)\right|<C^{|\alpha|}(\alpha !) b^{\mu_{n}} \sup _{x \in K}|P(x)|
$$

We have the following result:

Theorem (I.2). Let $K$ be a compact set in $\mathbf{R}^{N}$ and $\left(H_{n}\right)$ and $\left(\mu_{n}\right)$ satisfy (I.3) and (I.4). The properties (A), (B) and (M) are equivalent.

(3) If $\alpha=\left(\alpha_{1}, \ldots, \alpha_{N}\right) \in N^{N}$, we denote $|\alpha|=\alpha_{1}+\cdots+\alpha_{N}, \alpha !=\left(\alpha_{1} !\right) \cdots\left(\alpha_{N} !\right), D^{\alpha}$ $=\left(\partial / \partial x_{1}\right)^{\alpha_{1}} \cdots\left(\partial / \partial x_{N}\right)^{\alpha_{N}}$. 
By the virtue of Theorem (I.1), it is sufficient to show that (M) and (B) are equivalent. We leave this proof to the reader; we just observe that "(B) implies (M)" follows from the Cauchy integral formula, and "(M) implies (B)" uses a suitable Taylor expansion.

Let us point out that if $\mathcal{N}(K)=0$ (i.e. no component of $K$ is contained in an analytic set in $\mathbf{R}^{N}$ ), the properties (B) and (M) may be simplified since we have $Q=0$ in (I.6) and (I.15). This case deserves more attention.

$K,\left(H_{n}\right)$ and $\left(\mu_{n}\right)$ being given we have:

Theorem (1.3). Let $K$ satisfy $\mathcal{N}(K)=0$. Property (B) holds for $K$ if and only if, for any $x \in K$ there exists a compact set $L_{x}$ in $\mathbf{R}^{N}$ satisfying

(I.16) $x \in L_{x}, L_{x} \subset K, \mathcal{N}\left(L_{x}\right)=0$ and (B) holds for $L_{x}$.

Proof. It is clear that (B) (for $K$ ) implies the property asserted in the theorem. Let us prove the converse.

The assumption is that for any $x \in K$, there exists $L_{x}$ which satisfies (I.16). Therefore, for $b>1$, there exists $\Omega_{x}$, open set in $C^{N}$, neighborhood of $L_{x}$, such that for any $P \in H_{n}$

$$
\sup _{y \in \Omega_{x}}|P(y)| \leq b^{\mu_{n}} \sup _{y \in L_{x}}|P(y)| .
$$

Let us denote $\Omega=\cup_{x \in K} \Omega_{x} . \Omega$ is a neighborhood of $K$ in $\mathbf{C}^{N}$, and we have for any $P \in H_{n}$

$$
\sup _{y \in \Omega}|P(y)| \leq b^{\mu_{n}} \sup _{y \in K}|P(y)|
$$

which shows that (B) holds for $K$.

Remark (I.1). Without the assumption $\mathcal{N}\left(L_{x}\right)=0$, the preceding theorem would be false (see Example (II.2) below).

In property (B), is it possible to consider only the open set $\Omega$, neighborhood of $K$ in $\mathbf{R}^{N}$, instead of $\mathbf{C}^{N}$ ? Let us first introduce

Property (B'): Bernstein's inequality in the real domain. For any real number $b>1$, there exists an open set $V$, neighborhood of $K$ in $\mathbf{R}^{N}$, such that any $P \in H_{n}$ is analytic in $V$ and satisfies

$$
\sup _{x \in V}|P(x)| \leq b^{\mu_{n}} \sup _{x \in K}|P(x)|
$$

Now we have

Theorem (1.4). Let $K$ satisfy $\mathcal{N}(K)=0$, and for any point $x$ in $K$, there exists a fundamental system of compact neighborhoods of $x$ in $\mathbf{R}^{N}$ which satisfy (B). Then the properties $(\mathrm{B})$ and $\left(\mathrm{B}^{\prime}\right)$ are equivalent.

Proof. Since $\mathcal{N}(K)=0$, it is obvious that $(B)$ implies $\left(B^{\prime}\right)$. We shall prove the inverse implication. 
Let us assume $\left(B^{\prime}\right)$. We must show that for any $b>1$, there exists $\Omega$, open neighborhood of $K$ in $C^{N}$, such that any $P \in H_{n}$ is holomorphic in $\Omega$ and

$$
\sup _{x \in \Omega}|P(x)| \leq b^{\mu_{n}} \sup _{x \in K}|P(x)| \text {. }
$$

Let $V$ be the open set in $\mathbf{R}^{N}$ associated to $K$ by $\left(\mathrm{B}^{\prime}\right)$ with $b^{1 / 2}$ (instead of $b$ ). For any point $x \in K$, there exists a compact neighborhood $L_{x}$ contained in $V$ which satisfies (B). Therefore there exists $\Omega_{x}$, open neighborhood of $L_{x}$ in $C^{N}$, such that any $P \in H_{n}$ is holomorphic in $\Omega_{x}$ and

$$
\sup _{y \in \Omega_{x}}|P(y)| \leq b^{\mu_{n / 2}} \sup _{y \in L_{x}}|P(y)| .
$$

(I.19) together with

$$
\sup _{y \in L_{x}}|P(y)| \leq \sup _{y \in V}|P(y)| \leq b^{\mu_{n / 2}} \sup _{y \in K}|P(y)|
$$

gives us (I.18) with $\Omega=\cup_{x \in K} \Omega_{x}$, and ends the proof of Theorem (I.4).

\section{POLYNOMIAL APPROXIMATION OF ANALYTIC FUNCTIONS}

1. Application of part I and examples. Let $\mathscr{P}_{n}$ be the space of polynomials with complex coefficients in $N$ variables and with total degree $\leq n$.

We choose henceforth $H_{n}=\mathscr{P}_{n}$ and $\mu_{n}=n$.

Let us recall the following result (see [1]):

Let $K$ be a compact set in $\mathbf{R}^{N}$ and $f \in \mathcal{A}(K)$; there exists an $\left.a \in\right] 0,1[$ and $C>0$ such that, for any $n \in \mathbf{N}$,

$$
d_{k}\left(f ; \mathscr{P}_{n}\right) \leq C a^{n} .
$$

Hence, property (A), in this case $\left(\mathscr{P}_{n}=H_{n}\right.$ and $\left.\mu_{n}=n\right)$ may be formulated in the following way:

In order that a continuous function defined on $K$ be in $\mathcal{A}(K)$, it is necessary and sufficient that it be possible to find an $a \in] 0,1[$ and $C>0$ such that, for any $n \in N$, $d_{k}\left(f, \mathscr{P}_{n}\right) \leq C a^{n}$.

We also recall the following Bernstein result (see [2] and also [1]).

(II.2) Any closed, bounded parallelepiped in $\mathbf{R}^{N}$ whose interior is not empty satisfies (A).

We must point out that there exist some compact sets in $\mathbf{R}^{N}$ which do not satisfy (A). Here are two examples.

Example (II.1). For any strictly decreasing sequence $\left(x_{n}\right)$ of positive real numbers convergent to 0 the compact $K=\left\{x_{1}, \ldots, x_{n}, \ldots\right\} \cup\{0\}$ does not satisfy (A) in $\mathbf{R}$.

Proof. We use Theorem (I.1) and we show that $K$ does not satisfy (B). We prove this fact by contradiction.

Assume that (B) holds for $K$. Without loss of generality we may suppose $x_{1} \leq 1$. Let $b>1$. Since (B) is true, there exists $j \in \mathbf{N}$ such that, for any $P \in \mathscr{P}_{n}$ 
Let us set

$$
\sup _{x \in\left[0,\left(x_{j}+x_{j-1}\right) / 2\right]}|P(x)| \leq b^{n} \sup _{x \in K}|P(x)|\left({ }^{4}\right) .
$$

$$
P_{n}(x)=\left(x-x_{1}\right) \cdots\left(x-x_{n}\right), \quad a=\left(x_{j-1}-x_{j}\right) / 2 .
$$

We have, for each $n \in \mathbf{N}$.

$$
\begin{aligned}
\sup _{x \in\left[0,\left(x_{j}+x_{j-1}\right) / 2\right]}\left|P_{n}(x)\right| & \geq\left|P_{n}\left(\left(x_{j}+x_{j-1}\right) / 2\right)\right| \geq a^{n}, \\
\sup _{x \in K}\left|P_{n}(x)\right| & =x_{1} \cdots x_{n} .
\end{aligned}
$$

Finally, we get from (II.3)

$$
a^{n} \leq b^{n}\left(x_{1} \cdots x_{n}\right) \text { or } d^{n} \leq x_{1} \cdots x_{n}
$$

with $d=a / b$, which is impossible since $\left(x_{n}\right)$ is strictly decreasing, convergent to 0 and $d \in] 0,1[$.

Example (II.2). In $\mathbf{R}^{2}$, we denote

$$
\begin{aligned}
\Pi & =\left\{\left(x_{1}, x_{2}\right),-1 \leq x_{1} \leq+1,-1 \leq x_{2} \leq+1\right\}, \\
S & =\left\{\left(x_{1}, x_{2}\right), 1 \leq x_{1} \leq 2, x_{2}=0\right\} \\
K & =\Pi \cup S .
\end{aligned}
$$

Property (A) does not hold for $K$ in $\mathbf{R}^{2}$.

Proof. Let $f$ be defined on $K$ by

$$
\begin{aligned}
f(x) & =x_{2} /\left(x_{1}-2\right), & & \text { for } x \in \Pi, \\
& =0, & & \text { for } x \in S .
\end{aligned}
$$

It is obvious that $f \in C(K)$ and $f \notin A(K)$. However we can show that $f$ satisfies (II.1) with suitable $C>0$ and $a \in] 0,1[$. Indeed we have

$$
d_{K}\left(f, \mathscr{P}_{n}\right) \leq d_{\Pi}\left(f, y \mathscr{P}_{n-1}\right) \leq d_{\Pi}\left(1 /(x-2), \mathscr{P}_{n-1}\right) .
$$

The rest of the proof is easy.

Remark (II.1). It is false to say that the union or the intersection of two compact sets in $\mathbf{R}^{N}$ satisfying (A) must satisfy (A). This can be shown using the preceding example.

(4) In fact, there exists $\Omega$, neighborhood of $K$ in $C$, such that, for any $P \in \mathscr{P}_{R}$ there exists $Q \in \delta N_{K}(\Omega)$ satisfying $\sup _{x \in \Omega}|P(x)+Q(x)|<b^{n} \sup _{x \in K}|P(x)|$. Since $Q$ vanishes on $K$, and is analytic in $\Omega, Q$ must vanish on the component of 0 in $\Omega$ and hence vanishes on $\left[0,\left(x_{j}+x_{j-1}\right) / 2\right]$ for some $j \in \mathbf{N}$. 
We give now some examples of compact sets in $\mathbf{R}^{N}$ for which property (A) holds.

The following case is a simple application of Theorem (1.3) together with (II.2).

Example (11.3). Let $K$ be a compact set in $\mathbf{R}^{N}$ such that, for any $x \in K$, there exists a closed parallelepiped $\Pi_{x}$ with nonempty interior satisfying $x \in \Pi_{x}$ and $\Pi_{x} \subset K$. Then property (A) holds for $K$.

Here is another example.

Example (1I.4). Let $K$ be the compact set given in example (II.1). We define $K_{1}=K \cup[-1,0]$. The compact set $K_{1}$ satisfies (A) in $\mathbf{R}$.

Proof. We prove, actually, that $K_{1}$ satisfies (B). Let $b>1$. There exists $\Omega_{1}$ open set in $C$ such that for any $P \in \mathscr{P}_{n}$

$$
\sup _{x \in \Omega_{1}}|P(x)| \leq b^{n} \sup _{x \in[-1,0]}|P(x)| \text {. }
$$

Let $x_{1}, \ldots, x_{n_{0}}$ be the elements of the sequence $\left(x_{n}\right)$ which are not contained in $\Omega$. We choose $B_{1}, \ldots, B_{n_{0}}$, open balls in $\mathbf{C}$, pairwise disjoint and centered at $x_{1}, \ldots, x_{n_{0}}$ respectively. We set $\Omega=\Omega_{1} \cup B_{1} \cup \cdots \cup B_{n_{0}}$, and for $P \in \mathscr{P}_{n}$,

$$
\begin{aligned}
Q(x) & =0, & & \text { for } x \in \Omega_{1}, \\
& =P\left(x_{i}\right)-P(x), & & \text { for } x \in B_{i}, i=1, \ldots, n_{0} .
\end{aligned}
$$

We have

$$
\sup _{x \in \Omega}|P(x)+Q(x)| \leq b^{n} \sup _{x \in K_{1}}|P(x)|
$$

2. A sufficient criterion. We shall give here a sufficient criterion for a compact set $K$ in $\mathbf{R}^{N}$ in order to satisfy property (A). (This criterion is more general than the one given in Example (II.3).)

We introduce first some notations. Let $I=[A, B]$ be an interval in $\mathbf{R}^{N}$, (I is the set of points $t A+(1-t) B$ with $0 \leq t \leq 1)$. For any $h>1$, we denote by $I(h)$ the homothetic interval obtained from $I$ by the homothety centered at $(A+B) / 2$ and whose ratio of similitude is $h$.

If $K$ is a compact set in $\mathbf{R}^{N}$, we denote by $K(h)$ the union of $I(h)$ where $I$ is any interval contained in $K$.

$$
K(h)=\bigcup_{l \subset K} I(h)
$$

We say that $K$ satisfies the homothety criterion if, for any $h>1, K(h)$ is a neighborhood of $K$ in $\mathbf{R}^{N}$.

We have

Theorem (II.1). Property (A) holds for any compact set $K$ in $\mathbf{R}^{N}$ which satisfies the homothety criterion. 
Proof. It is easy to see that if $K$ satisfies the homothety criterion, we have $\mathcal{N}(K)=0$. Therefore, using Theorem (I.4), it is sufficient to prove that property (B') holds for such $K$.

From the classical Bernstein inequality on an interval (one variable, see [2] and [4]), we get for any $P \in \mathscr{P}_{n}$ and any $h>1$

$$
\sup _{x \in K(h)}|P(x)| \leq\left(h+\sqrt{\left(h^{2}-1\right)}\right)^{n} \sup _{x \in K}|P(x)| .
$$

Let $b>1$ be fixed. We choose $h$ such that

$$
h+\sqrt{\left(h^{2}-1\right)}=b \text {. }
$$

Since $K(h)$ is a neighborhood of $K$ in $\mathbf{R}^{N}$, property $\left(\mathrm{B}^{\prime}\right)$ holds for $K$.

Example (II.5). Let $\phi$ be a strictly increasing continuous function defined on $[0,1]$ with $\phi(0)=0$.

The compact set $K$ in $\mathbf{R}^{2}$ defined by $K=\left\{\left(x_{1}, x_{2}\right), 0 \leq x_{1} \leq 1,0 \leq x_{2} \leq \phi(x)\right\}$ satisfies the homothety criterion (and hence satisfies (A)).

Actually it is sufficient to prove that $(0,0)$ is an interior point of $K(h)$ for any $h>1$. We leave the proof for the reader.

Remark (II.2). With the notations of the preceding example, we choose

$$
\phi(x)=e^{-1 / x} \text { for } x \in[0,1] .
$$

Let us consider the function $f\left(x_{1}, x_{2}\right)=x_{2} / x_{1}$ defined on $K . f$ does not have a $c^{\infty}$ extension to any neighborhood of $K$ in $\mathbf{R}^{2}$ (since $\partial f / \partial x_{2}$ is not bounded!). However it is easy to see that there exist an $a \in] 0,1[$ and $C>0$ such that for any $n \in \mathbf{N}$

$$
d_{K}\left(f, \mathscr{P}_{n}\right) \leq C a^{n^{1 / 2}}
$$

This example is given in [7] to show that the rapid decrease of $d_{K}\left(f, \mathscr{P}_{n}\right)$ is not sufficient to conclude that $f \in C^{\infty}(K)$, in this case. It is interesting to note that this same compact does satisfy (A).

\section{BIBLIOGRAPHY}

1. M. S. Baouendi and C. Goulaouic, Approximation polynomiale de fonctions $C^{\infty}$ et analytiques, Ann. Inst. Fourier (Grenoble) 21 (1971), 149-173.

2. S. Bernstein, Collected works, Izdat. Akad. Nauk SSSR, Moscow, 1952. (Russian) MR 14, 2.

3. A Grothendieck, Espaces vectoriels topologiques, Instituto de Mathemática Pura e Aplicada, Universidade de São Paulo, São Paulo, 1954. MR 17, 1110.

4. G. G. Lorentz, Approximation of functions, Holt, Rinehart and Winston, New York, 1966. MR 35 \#4642.

5. S. N. Mergelian, Uniform approximations to functions of a complex variable, Uspehi Mat. Nauk 7 (1952), no. 2 (48), 31-122; English transl., Amer. Math. Soc. Transl. (1) 3 (1962), 294-391. MR 14, 547. 
6. J. L. Walsh, Interpolation and approximation by rational functions in the complex domain, 4th ed., Amer. Math. Soc. Colloq. Publ., vol. 20, Amer. Math. Soc., Providence, R.I., 1965. MR 36 \#1672b.

7. M. Zerner, Développement en série de polynomes orthogonaux des fonctions indéfiniment différentiables, C. R. Acad. Sci. Paris Sér. A-B 268 (1969), A218-A220. MR 40 \#717.

Department of Mathematics, Purdue University, Lapayette, Indiana 47907

Département de Mathématique, Centre D’Orsay, Université de Paris-Sud, Paris, France 